\title{
SOME INTEGRAL CHARACTERIZATIONS OF ABSOLUTE CONTINUITY
}

\author{
WILIIAM D. I.. APPLING
}

1. Introduction. Suppose $F$ is a field of subsets of a set $U, R$ is the set of all real-valued functions on $F, R_{B}$ is the set of all bounded elements of $R, R^{+}$is the set of all nonnegative-valued elements of $R, R_{B}^{+}$ $=R_{B} \cap R^{+}$, and $R_{A}^{+}$is the set of all finitely additive elements of $R^{+}$.

In a previous paper [3] the author has proved the following characterization theorem for absolute continuity:

THEOREM A. If each of $s$ and $t$ is a real-valued nondecreasing function on the number interval $[a, b]$, then $s$ is absolutely continuous with respect to $t$ iff for every real-valued, bounded function $P$ of subintervals of $[a, b]$ for which the integral (see [3] or $\$ 2$ of this paper) $\int_{[a, b]} P(I) d$ t exists, the integral $\int_{[a, b]} P(I) d s$ exists.

In this paper we prove an extension of Theorem A, giving a necessary and sufficient condition that the above type of absolute continuity characterization holds with respect to the elements of $R_{A}^{+}$and $R_{B}^{+}$. Specifically, we prove $(\$ 5)$ the following theorem:

THEOREM 5.1. The following two statements are equivalent:

(1) If $q$ is in $R_{A}^{+}$and $q(U)>0$, then there is an element $T$ of $R_{B}^{+}$such that the integral $(\$ 2) \int_{U} T(I) q(I)$ does not exist.

(2) If each of $h$ and $m$ is in $R_{A}^{+}$, then $h$ is absolutely continuous with respect to $m$ iff for each $P$ in $R_{B}^{+}$such that $\int_{U} P(I) m(I)$ exists, $\int_{U} P(I) h(I)$ exists.

Prior to proving Theorem 5.1, we prove ( $\$ 4$ ) the following theorem, of which Theorem 5.1 is a consequence:

THEOREM 4.1. If each of $h$ and $m$ is in $R_{A}^{+}$, then the following four statements are equivalent:

(1) If $P$ is in $R_{B}^{+}$and $\int_{U} P(I) m(I)=0$ and $\int_{U} P(I) h(I)$ exists, then $\int_{U} P(I) h(I)=0$.

(2) If $Q$ is in $R_{B}^{+}$and for some $K>0, Q(I)[\max \{Q(I), K\}-Q(I)]$ $=0$ for all $I$ in $F$ and $\int_{U} Q(I) m(I)=0$ and $\int_{U} Q(I) h(I)$ exists, then $\int_{U} Q(I) h(I)=0$.

(3) If $T$ is in $R_{B}^{+}$and $T(I)[T(I)-1]=0$ for all $I$ in $F$ and $\int_{U} T(I) m(I)$ $=0$ and $\int_{U} T(I) h(I)$ exists, then $\int_{U} T(I) h(I)=0$.

(4) $h$ is absolutely continuous with respect to $\mathrm{m}$.

Received by the editors December 16, 1965. 
2. Preliminary theorems and definitions. If $V$ is in $F$, then the statement that $D$ is a subdivision of $V$ means that $D$ is a finite collection of mutually exclusive sets of $F$ whose union is $V$.

If $D$ is a subdivision of a set $V$ of $F$, then the statement that $E$ is a refinement of $D$ means that $E$ is a subdivision of $V$ such that every set of $E$ is a subset of some set of $D$.

Throughout this paper all integrals discussed will be Hellinger [5] type limits (i.e., for refinements of subdivisions) of the appropriate sums. Thus, if $H$ is in $R$, then $\int_{U} H(I)$ exists iff for each $V$ in $F$, $\int_{V} H(I)$ exists, in which case the function $M$ on $F$ defined by $M(V)$ $=\int_{V} H(I)$ is finitely additive.

We state without proof a theorem of Kolmogoroff [6].

Theorem 2.K.1. If $H$ is in $R$ and $\int_{U} H(I)$ exists, then $\int_{U} \mid H(I)$ $-\int_{I} H(J) \mid=0$.

We state without proof an immediate corollary to Theorem 2.K.1.

Corollary 2.K.1. If $P$ is in $R_{B}$ and $Q$ is in $R$ and $\int_{U} Q(I)$ exists, then $\int_{U}|P(I)|\left|Q(I)-\int_{I} Q(J)\right|=0$, so that if $V$ is in $F$, then $\int_{V} P(I) Q(I)$ exists iff $\int_{V} P(I) \int_{I} Q(J)$ exists, in which case equality holds.

We also state a lemma without proof.

Lemma 2.1. If $P$ is in $R_{B}$ and $Q$ is in $R$ and for each $c>0$ there is a $T$ in $R$ and a subdivision $D$ of $U$ such that $\sum_{E}|T(I)-Q(I)|<c$ for all refinements $E$ of $D$ and $\int_{U} P(I) T(I)$ exists, then $\int_{U} P(I) Q(I)$ exists.

We end this section by stating without proof an extension of a previous interval function theorem of the author [1]. The proof carries over for the appropriate elements of $R$ with only minor modifications.

Theorem 2.A.1. If each of $P$ and $Q$ is in $R_{B}$ and $w$ is in $R_{A}^{+}$and each of $\int_{U} P(I) w(I)$ and $\int_{U} Q(I) w(I)$ exists, then $\int_{U} P(I) Q(I) w(I)$ exists.

3. The integral $\int_{V} \min \{X(I), Y(I)\}$. Suppose each of $a, b, c$ and $d$ is a number. We see that $\min \{a, c\}+\min \{b, d\} \leqq \min \{a+b, c+d\}$ $\leqq \max \{a+b, c+d\} \leqq \max \{a, c\}+\max \{b, d\}$. This implies that if each of $x$ and $y$ is in $R_{A}^{+}$, and $E$ is a refinement of the subdivision $D$ of the set $V$ of $F$, then $0 \leqq \sum_{E} \min \{x(I), y(I)\} \leqq \sum_{D} \min \{x(I), y(I)\}$ $\leqq \sum_{D} \max \{x(I), \quad y(I)\} \leqq \sum_{E} \max \{x(I), \quad y(I)\} \leqq x(V)+y(V), \quad$ so that each of $\int_{V} \min \{x(I), y(I)\}$ and $\int_{V} \max \{x(I), y(I)\}$ exists.

We also see that $\max \{|\min \{a, c\}-\min \{b, d\}|, \mid \max \{a, c\}$ $-\max \{b, d\} \mid\} \leqq|a-b|+|c-d|$. This, together with Theorem 2.K.1, implies the following theorem which we state without proof: 
THEOREM 3.K.1. If each of $P$ and $Q$ is in $R^{+}$and each of $\int_{U} P(I)$ and $\int_{U} Q(I)$ exists, then

$$
\begin{aligned}
\int_{U} \max \{\mid \min & \left\{\int_{I} P(J), \int_{I} Q(J)\right\}-\min \{P(I), Q(I)\} \mid, \\
& \left.\left|\max \left\{\int_{I} P(J), \int_{I} Q(J)\right\}-\max \{P(I), Q(I)\}\right|\right\}=0,
\end{aligned}
$$

so that, if $V$ is in $F$, then each of $\int_{V} \min \{P(I), Q(I)\}$ and $\int_{V} \max \{P(I)$, $Q(I)\}$ exists and equals, respectively, $\int_{V} \min \left\{\int_{I} P(J), \int_{I} Q(J)\right\}$ and $\int_{V} \max \left\{\int_{I} P(J), \int_{I} Q(J)\right\}$.

Throughout this paper we shall of ten simply write in tegrals whose existence or equivalence to integrals is a simple consequence of the preceding statements in $\S \S 2$ and 3 , and leave the proof of existence or equivalence to the reader.

We state a previous theorem of the author [4].

Theorem 3.A.1. If each of $P$ and $Q$ is in $R^{+}$and $\int_{U} \min \{P(I), Q(I)\}$ $=0$, and $0 \leqq K$, then $\int_{U} \min \{P(I), K Q(I)\}=0$.

Suppose each of $m$ and $h$ is in $R_{A}^{+}$and that for each $V$ in $F$

$$
g(V)=\sup \int_{V} \min \{h(I), K m(I)\} \quad \text { for } 0<K .
$$

We see that each of $g$ and $h-g$ is in $R_{A}^{+}$.

Suppose each of $r$ and $h-r$ is in $R_{A}^{+}$.

We state two previous theorems of the author [2] without proof.

THEOREM 3.A.2. $\int_{U} \min \{h(I)-r(I), m(I)\}=0$ iff $r-g$ is in $R_{A}^{+}$.

THEOREM 3.A.3. $r$ is absolutely continuous with respect to $m$ iff $g-r$ is in $R_{A}^{+}$.

We end this section by proving a lemma which we will need to prove Theorem 4.1 .

Lemma 3.1. If $1 \leqq K$, then $\int_{V} \min \{K[h(I)-g(I)], h(I)\}=h(V)$ $-g(V)$ for each $V$ in $F$.

Proof. Let $k$ be the element of $R_{A}^{+}$defined by $k(V)=\int_{V} \min \{K[h(I)$ $-g(I)], h(I)\}$.

Let $r=h-k$. Each of $r$ and $h-r$ is in $R_{A}^{+} \cdot \int_{U} \min \{m(I), h(I)-r(I)\}$ $=\int_{U} \min \{m(I), k(I)\} \leqq \int_{U} \min \{m(I), K[h(I)-g(I)]\}=0$, by Theorems 3.A.2 and 3.A.1. It follows from Theorem 3.A.2 that $r-g$ is in $R_{A}^{+}$, so that $(h-g)-k$ is in $R_{A}^{+}$. 
Therefore, if $V$ is in $F$, then $k(V) \leqq h(V)-g(V)=\int_{V} \min \{h(I)$ $-g(I), h(I)\} \leqq \int_{V} \min \{K[h(I)-g(I)], h(I)\}=k(V)$, so that $h(V)$ $-g(V)=k(V)=\int_{V} \min \{K[h(I)-g(I)], h(I)\}$.

4. A zero-valued integral characterization of absolute continuity. We adopt the convention that if each of $a$ and $b$ is a number, then $a / b=0$ if $b=0$, and has the usual meaning otherwise.

We now prove Theorem 4.1, as stated in the introduction.

Proof of Theorem 4.1. We first show that (4) implies (1). Suppose (4) is true and $Q$ is an element of $R_{B}^{+}$such that $\int_{U} Q(I) m(I)=0$. If $0<K$, then $\int_{U} Q(I) \min \{K, h(I) / m(I)\} m(I)$ exists by Theorem 2.A. 1 and is $\int_{U} Q(I) \int_{I} \min \{K m(J), \quad h(J)\} \leqq \int_{U} K Q(I) m(I)=0$. By Theorem 3.A.3, $\int_{U}\left|h(I)-\int_{I} \min \{h(J), K m(J)\}\right| \rightarrow 0$ as $K \rightarrow \infty$, so that by Lemma 2.1, $\int_{U} Q(I) h(I)$ exists. Furthermore, $\int_{U} Q(I) h(I)$ $=\int_{U} Q(I) h(I)-0=\int_{U} Q(I) h(I)-\int_{U} Q(I) \min \{K m(I), h(I)\}$ $=\int_{U} Q(I)\left[h(I)-\int_{I} \min \{K m(J), h(J)\}\right] \leqq M \int_{U} \mid h(I)-\int_{I} \min \{K m(J)$, $h(J)\} \mid \rightarrow 0$ as $K \rightarrow \infty$, where $Q(I) \leqq M$ for all $I$ in $F$. Therefore $\int_{U} Q(I) h(I)=0$.

Therefore (4) implies (1).

It is obvious that (1) implies (2) and (2) implies (3).

We now prove that (3) implies (2). Suppose (3) is true and $Q$ is in $R_{B}^{+}$, for some $K>0, Q(I)\lfloor\max \{Q(I), K\}-Q(I)]=0$ for all $I$ in $F$, $\int_{U} Q(I) m(I)=0$ and $\int_{U} Q(I) h(I)$ exists. For each $I$ in $F$, let $T(I)$ $=(1 / K) \min \{Q(I), K\}$. We see that for each $I$ in $F, T(I)[T(I)-1]$ $=0$. Now $0=\int_{U}(1 / K) \min \left\{\int_{I} Q(J) m(J), K m(I)\right\}=\int_{U}(1 / K) \min \{Q(I)$, $K\} m(I)=\int_{U} T(I) m(I)$. Furthermore, $\int_{U}(1 / K) \min \left\{\int_{I} Q(J) h(J)\right.$, $K h(I)\}=\int_{U}(1 / K) \min \{Q(I), K\} h(I)=\int_{U} T(I) h(I)$. Therefore $0=$ $\int_{U} T(I) h(I)=\int_{U}(1 / K) \min \{Q(I), K\} h(I)$, so that $0=\int_{U} \min \{Q(I)$, $K\} h(I)$, which implies by Theorem 3.A.1 that $0=\int_{U} \min \{Q(I), M\} h(I)$ $=\int_{U} Q(I) h(I)$, where $Q(I) \leqq M$ for all $I$ in $F$.

Therefore (3) implies (2).

We now prove that (2) implies (4). Suppose (2) is true. For each $V$ in $F$, let $h^{*}(V)=\int_{V} \max \{h(I), m(I)\}$ and $g^{*}(V)=\sup \int_{V} \min \left\{h^{*}(I)\right.$, $K m(I)\}$ for $0<K$.

Suppose $Q$ is in $R_{B}^{+}, \int_{U} Q(I) m(I)=0$, and $\int_{U} Q(I) h^{*}(I)$ exists. By Theorem 2.A.1, $\int_{U} Q(I)\left[h(I) / h^{*}(I)\right] h^{*}(I)$ exists and is $\int_{U} Q(I) h(I)=0$. Therefore $\int_{U} Q(I) h^{*}(I)=\int_{U} \max \left\{\int_{I} Q(J) m(J), \int_{I} Q(J) h(J)\right\}=0$.

By Lemma 3.1, $\int_{V} \min \left\{2\left[h^{*}(I)-g^{*}(I)\right], h^{*}(I)\right\}=h^{*}(V)-g^{*}(V)$ for all $V$ in $F$.

Let $Q$ be the element of $R$ defined by

$$
\begin{aligned}
& Q(I)=0 \quad \text { if }\left[h^{*}(I)-g^{*}(I)\right] / h^{*}(I)<1 / 2, \\
& Q(I)=\left[h^{*}(I)-g^{*}(I)\right] / h^{*}(I) \quad \text { otherwise. }
\end{aligned}
$$


We see that $Q$ is in $R_{B}^{+}$and that for each $I$ in $F, Q(I)[\max \{Q(I), 1 / 2\}$ $-Q(I)]=0$.

Suppose $c>0$ and $V$ is in $F$. By Lemma 3.1 there is a subdivision $D$ of $V$ such that if $E$ is a refinement of $D$, then

$$
\begin{aligned}
c> & \sum_{E}\left(\min \left\{2\left[h^{*}(I)-g^{*}(I)\right], h^{*}(I)\right\}-\left[h^{*}(I)-g^{*}(I)\right]\right) \\
= & \sum_{E^{*}}\left(2\left[h^{*}(I)-g^{*}(I)\right]-\left[h^{*}(I)-g^{*}(I)\right]\right) \\
& +\sum_{E-E^{*}}\left(h^{*}(I)-\left[h^{*}(I)-g^{*}(I)\right]\right)=\sum_{E^{*}}\left[h^{*}(I)-g^{*}(I)\right] \\
& +\sum_{E-E^{*}} g^{*}(I) \geqq \sum_{E^{*}}\left[h^{*}(I)-g^{*}(I)\right],
\end{aligned}
$$

where $E^{*}$ is the set (if any) of all $I$ in $E$ such that

$$
\begin{aligned}
& {\left[h^{*}(I)-g^{*}(I)\right] / h^{*}(I)<\frac{1}{2} \text {, so that }} \\
& \left|h^{*}(V)-g^{*}(V)-\sum_{E} Q(I) h^{*}(I)\right|=\mid \sum_{E^{*}}\left[h^{*}(I)-g^{*}(I)-Q(I) h^{*}(I)\right] \\
& +\sum_{E-E^{*}}\left[h^{*}(I)-g^{*}(I)-Q(I) h^{*}(I)\right] \\
& =\left|\sum_{E^{*}}\left[h^{*}(I)-g^{*}(I)\right]+\sum_{E-E^{*}}\left[h^{*}(I)-g^{*}(I)-\left\{h^{*}(I)-g^{*}(I)\right\}\right]\right| \\
& =\sum_{E^{*}}\left[h^{*}(I)-g^{*}(I)\right]<c .
\end{aligned}
$$

Therefore, for each $V$ in $F, h^{*}(V)-g^{*}(V)=\int_{V} Q(I) h^{*}(I)$.

We see that for each $V$ in $F, Q(V) \leqq 1$ and $Q(V) m(V) \leqq h^{*}(V)$ $-g^{*}(V)$.

Now, if $D$ is a subdivision of $U$, then $\sum_{D} Q(I) m(I)=\sum_{D} \min \{1$, $Q(I)\} m(I)=\sum_{D} \min \{m(I), Q(I) m(I)\} \leqq \sum_{D} \min \left\{m(I), h^{*}(I)\right.$ $\left.-g^{*}(I)\right\}$, so that by Theorem 3.A.2, $\int_{U} Q(I) m(I)=0$.

Therefore, $0=\int_{U} Q(I) h^{*}(I)=h^{*}(U)-g^{*}(U) ; h^{*}$ is $g^{*}$, so that, by Theorem 3.A.3, $h^{*}$ is absolutely continuous with respect to $m$. Since $h^{*}-h$ is in $R_{A}^{+}, h$ is absolutely continuous with respect to $m$.

Therefore (2) implies (4).

Therefore (1), (2), (3) and (4) are equivalent.

5. An integrability characterization of absolute continuity. In this section we prove Theorem 5.1, as stated in the introduction.

Proof of Theorem 5.1. We first show that (1) implies (2). Suppose (1) is true and each of $h$ and $m$ is in $R_{A}^{+}$. 
Suppose $h$ is absolutely continuous with respect to $m$ and $Q$ is in $R_{B}^{+}$and $\int_{U} Q(I) m(I)$ exists. If $0<K$, then by Theorem 2.A.1, $\int_{U} Q(I) \min \{K, h(I) / m(I)\} m(I)$ exists and is $\int_{U} Q(I) \int_{I} \min \{K m(J)$, $h(J)\}$. It therefore follows from Theorem 3.A.3 and Lemma 2.1 that $\int_{U} Q(I) h(I)$ exists.

Now suppose that if $Q$ is in $R_{B}^{+}$and $\int_{U} Q(I) m(I)$ exists, then $\int_{{ }^{r}} Q(I) h(I)$ exists.

We show that if $P$ is in $R_{B}^{+}, \int_{U} P(I) m(I)=0$ and $\int_{U} P(I) h(I)$ exists, then $0=\int_{U} P(I) h(I)$. Suppose that for some $P$ in $R_{B}^{+}, 0=\int_{U} P(I) m(I)$ and $\int_{U} P(I) h(I)$ exists, but $0<\int_{U} P(I) h(I)$. Let $q$ be the element of $R_{A}^{+}$defined by $q(V)=\int_{V} P(I) h(I)$. Since $q(U)>0$, it follows that there is a $W$ in $R_{B}^{+}$such that $\int_{U} W(I) q(I)$ does not exist, so that by Corollary 2.K.1, $\int_{U} W(I) P(I) h(I)$ does not exist.

There is a number $M$ such that $W(I) \leqq M$ for all $I$ in $F$, so that if $D$ is a subdivision of $U$, then $\sum_{D} W(I) P(I) m(I) \leqq M \sum_{D} P(I) m(I)$, so that $0=\int_{U}[W(I) P(I)] m(I)$, which implies that $\int_{U}[W(I) P(I)] h(I)$ exists, a contradiction.

It therefore follows from Theorem 4.1 that $h$ is absolutely continuous with respect to $m$.

Therefore (1) implies (2).

We now show that (2) implies (1). Suppose (2) is true and $q$ is in $R_{A}^{+}$and $q(U)>0$. Obviously $q$ is not absolutely continuous with respect to the element $O$ of $R_{A}^{+}$defined by $O(I)=0$. Therefore there is a $P$ in $R_{B}^{+}$such that $\int_{U} P(I) O(I)$ exists, but such that $\int_{U} P(I) q(I)$ does not exist.

Therefore (2) implies (1).

Therefore (1) and (2) are equivalent.

\section{REFERENCES}

1. W. D. L. Appling, Interval functions and the Hellinger integral, Duke Math. J. 29 (1962), 515-520.

2. - Characterizations of some absolutely continuous additive set functions, Rend. Circ. Mat. Palermo (2) 12 (1963), 347-352.

3. - Interval functions and absolute continuity, Illinois J. Math. 8 (1964), $439-445$.

4. - A uniqueness characierization of absolute continuity, Monatsh. Math. 70 (1966), 97-105.

5. E. Hellinger, Die orthogonalinvarianten quadratischer Formen von unendlichvielen Variablen, Dissertation, Göttingen, 1907.

6. A. Kolmogoroff, Untersuchungen über den Integralbegriff, Math. Ann. 103 (1930), 654-696.

North Texas State University 